\title{
Stellar dynamics and feedback connections to stellar evolution
}

\author{
Nataliya M. Ivanova \\ Canadian Institute for Theoretical Astrophysics, University of Toronto, \\ 60 St. George Street, Toronto, ON M5S 3H8, Canada \\ email: nata@cita.utoronto.ca
}

\begin{abstract}
In dense stellar systems, dynamical interactions between objects inevitably lead to frequent formation of exotic stellar objects and multiple systems, thereby imposing new questions for the stellar evolution theory. The evolutionary path of such systems could be different from that of the unperturbed objects, therefore, we must re-evaluate their evolutionary treatment to clarify their consequent dynamical evolution. We review briefly the classes of important dynamical encounters and discuss several post-encounter outcomes that may require more detailed attention or development of a new treatment in stellar evolution: evolution of complex merger products, spun-up stars, binaries with stripped giants and triples.
\end{abstract}

Keywords. globular clusters: general, stars: evolution

Let us examine encounters between objects in the order of increasing distance of closest approach. In the smallest limit, an encounter leads to a head-on collision. The result of such event depends on the relative velocity at infinity $v_{\infty}$ and on the binding energies of the participating objects. It may lead either to a complete merger of the two objects, to a significant mass-loss or, in the case of collisions with giants, to a binary formation. As the distance increases, participants of the encounters still can be destroyed due to strong tidal forces. When the pericenter of an encounter is far enough for stars not to be destroyed through tidal interactions, but tidal forces are still very strong, binary formation may occur - a so-called tidal capture. However, if $v_{\infty}$ is large, the total kinetic energy that is needed to be dumped in stars by tidal interactions to form a bound system, becomes comparable to the binding energy of the participants, hence the tidal capture regime becomes unviable, and an encounter may lead only to the envelope spin up.

Among all the varieties of possible physical collisions and their outcomes, most well studied are collisions between two main sequence (MS) stars. They are popular as they lead to the formation of blue stragglers, a class of objects well distinguished observationally. Recent progress in the subject includes the automatizing of the evolutionary calculation of the merger product (Glebbeek \& Pols, in prep). However, this does not yet take into account the rotation of the merger product, and the rotation affects strongly the appearance (luminosity) and the evolutionary life-time of a blue straggler (Sills et al. 2001). Compared to collisions of MS stars, very little is done on what occurs in the collisions of more evolved stars. These are not rare: we find that at least $5 \%$ of all red giants present in the globular clusters (GCs) cores at $\sim 10$ Gyr have experienced collisions that resulted either in a significant increase of the envelope mass, or in the formation of hybrid core (where, in comparison to a normal AGB core, very massive He shell of $0.1-0.4$ $\mathrm{M}_{\odot}$ sits on the $\mathrm{CO}$ core). The evolution of such objects has not been studied within the standard stellar evolution.

Another outcome of a physical collision with a giant is the formation of an eccentric binary. The stripped giant core retains bound remnant envelope up to $0.1 \mathrm{M}_{\odot}$ (Lombardi et al. 2006). An envelope of such mass rebounds to its pre-collision size on a thermal 
time-scale. In addition, the mass of the envelope is not large enough to tidally circularize binary on this time-scale. As a result, the mass transfer may start in a non-circularized binary with rapidly expanding giant envelope.

When a strong tidal interaction results in a binary formation, it is important to clarify where and how much energy is deposited in the star, as the depth and the quantity affect the star expansion and the time-scales on which star will expand and contract (the latter is about of a thermal time-scale of the expanded star). Detailed stellar evolution with proper energy input should show whether it is possible to form a tight binary, or star will remain expanded too long compared to the time-scale of the tidal orbital decay. Special case is a fly-by encounter when neither a bound binary was formed nor destruction nor significant mass loss has occurred, but significant amount of the angular momentum was transferred to stars. The efficiency of the spin-up increases with $v_{\infty}$, and therefore any effect associated with extra mixing possible in fast rotating giants (see, e.g., Sweigart 1997; Denissenkov et al. 2006) will manifest stronger in clusters with bigger velocity dispersion.

Last, we want to emphasize the importance of triples evolution in GCs. Observations of field population reveal that significant fraction of stars are members of hierarchical systems of three and higher multiplicity Tokovinin 2001), and the fraction of triples is increasing when one considers short-periodic binaries (Pribulla \& Rucinski 2006) or massive stars (Zinnecker 2006 Zinnecker 2006). In addition to primordial triple population, binary-binary encounters frequently lead to the formation of triple hierarchical systems. The rate of their formation at 10-11 Gyrs is so high that up to $5 \%$ of all binaries have had participated in the triple formation during 1 Gyr (Ivanova 2006). Triples formation and evolution should lead to the modification of the binary population. Indeed, we find that the Kozai Mechanism (KM, Kozai 1962) will act in at least $30 \%$ of all dynamically formed triples. KM leads to the angular momentum transfer between the outer orbit to the inner one, resulting in the oscillations in the eccentricity of the inner orbit and the inclination angle between the two orbits. If an affected by KM triple has the characteristic time-scale between two successful collisions longer than its Kozai cycle, the inner binary can merge or set on the mass transfer before next encounter (Eggleton \& Kiseleva-Eggleton 2001, 2006), therefore triples should affect, e.g., the formation and evolution of X-ray binaries in GCs. This suggestion is also supported by results of our simulations where we find that the relative fraction of compact objects in the population of inner binaries of dynamically formed triples is two times larger than in the population of all the binaries.

\section{References}

Denissenkov, P. A., \& Chaboyer, B., Li, K. 2006, ApJ, 641, 1087

Eggleton, P. P., \& Kiseleva-Eggleton, L. 2006, in: S. Hubrig \& A. Tokovinin (eds.), Multiple Stars across the H-R Diagram, ESO Proc. (in press)

Eggleton, P. P., \& Kiseleva-Eggleton, L. 2001, ApJ 562, 1012

Ivanova, N. 2006 in: S. Hubrig \& A. Tokovinin (eds.), Multiple Stars across the H-R Diagram, ESO Proc., in press

Kozai, Y. 1962, AJ, 67, 591

Lombardi, J. C., Proulx, Z. F., Dooley, K. L., et al. 2006, ApJ 640, 441

Pribulla, T., \& Ruciknski S. 2006, AJ, 131, 2986

Sills, A. I., Faber, J. A., Lombardi, J. C., Rasio, F. A., \& Warren, A. R 2001, ApJ, 548, 323

Sweigart, A. V. 1997 ApJ (Letters), 474, L23

Tokovinin, A. A. 2001, in: H. Zinnecker \& R. D. Mathieu (eds.), The Formation of Binary Stars, Proc. IAU Symp. No. 200 (San Francisco: ASP), p. 84

Zinnecker, H. 2006, in: S. Hubrig \& A. Tokovinin (eds.), Multiple Stars across the H-R Diagram, ESO Proc., in press 